\title{
Polarization in Photodisintegration of the Deuteron
}

\author{
R. Gilman* \\ for the Jefferson Lab Hall A Collaboration \\ ${ }^{*}$ Rutgers University ${ }^{1}$ \\ Piscataway, New Jersey 08855 USA, and \\ Thomas Jefferson National Accelerator Laboratory ${ }^{2}$ \\ Newport News, Virginia 23606 USA
}

\begin{abstract}
Recoil proton polarization was measured in deuteron photodisintegration at $\theta_{\mathrm{cm}}=90^{\circ}$ for photon energies up to $2.4 \mathrm{GeV}$. The induced polarization $p_{y}$ is consistent with vanishing above $1 \mathrm{GeV}$, in surprising disagreement with meson-baryon calculations. From pQCD it is generally expected that hadron helicity is conserved, but, the polarization transfer observables do not vanish. As the data are inconsistent with existing meson baryon model predictions, and with expectations from $\mathrm{pQCD}$, we suggest that nonperturbative quark models will be needed to understand the underlying reaction dynamics.
\end{abstract}

\section{INTRODUCTION}

Whether a meson baryon or a quark gluon picture is more appropriate to describe high momentum transfer exclusive reactions is a fundamental issue in nuclear physics. It is often suggested that, at high momentum transfers / short distances, conventional meson baryon models will break down, and quark models will be needed. While self-consistent perturbative quantum chromodynamics (pQCD) calculations are believed to not generally describe exclusive reactions at currently accessible kinematics, various nonperturbative quark model approaches have been suggested.

The issue has been studied in several electromagnetic exclusive reactions at large momentum transfer, including deuteron photodisintegration [1], elastic electron deuteron scattering [2,3], and threshold deuteron electrodisintegration [4]. To date, the evidence is that meson baryon models work well, although sometimes the underlying reaction dynamics are ambiguous, as meson baryon and quark models give

1) Supported by U.S. National Science Foundation grant PHY 9803860

2) Southeastern Universities Research Association (SURA) manages the Thomas Jefferson National Accelerator Facility under DOE contract DE-AC05-84ER40150.

CP570, SPIN 2000, $14^{\text {th }}$ International Spin Physics Symposium, edited by K. Hatanaka et al. (C) 2001 American Institute of Physics 0-7354-0008-3/01/\$18.00 
similar results. For example, in elastic ed scattering, polarization measurements [3] show that only meson baryon models can describe the data well up to $Q^{2}=$ $1.7 \mathrm{GeV}^{2}$, while cross section measurements at higher momentum transfer [2] are consistent with both meson baryon calculations and quark models.

Similarly, in deuteron photodisintegration, the highest energy cross sections show an $s^{-11}$ scaling behavior at $\theta_{\mathrm{cm}}=90^{\circ}$ that is consistent with a meson baryon calculation [5], a nonperturbative QCD model [6], and pQCD [7]. The onset of the scaling is at $-t \approx 1 \mathrm{GeV}^{2}$, which corresponds to a spatial resolution of $\approx 0.2 \mathrm{fm}$, and to a momentum transfer $Q^{2}$ of nearly $4 \mathrm{GeV}^{2}$ in ed elastic scattering [8].

Thus, we have measured reoil proton polarizations, with circularly polarized beam, to provide data that may be better able to discriminate among the various models that appear to explain the cross section measurements. We extend the range of induced polarization measurements from a maximum energy of near $1 \mathrm{GeV}$ to about $2.4 \mathrm{GeV}$. We also present the first results for the double-polarization observables with circularly polarized beam and recoil proton polarization. Predictions for these observables are available from pQCD, and several meson baryon calculations of the induced polarization exist [9-13] - though only [12] extends the polarization predictions to energies above $1 \mathrm{GeV}$. Before the experiment ran, the general expectation was that $\mathrm{pQCD}$ predicts that the induced polarization vanishes, while the meson baryon calculations predict that resonance contributions lead to an induced polarization that is generally large and strongly energy dependent. Calculations are also underway in nonperturbative quark models $[14,15]$.

In $\mathrm{pQCD}$, vector interactions conserve quark helicity; to the extent that orbital angular momentum effects can be neglected, quark helicity conservation then leads to hadron helicity conservation (HHC) [16]. (In contrast, there are recent arguments for hadronic reactions that quark exchange diagrams will lead to violation of $\mathrm{HHC}[17]$.) $\mathrm{HHC}$ requires that the sum of the components of the hadronic spins along their respective momentum directions is the same for both initial and final states, and gives predictions for some spin observables. In particular, the recoil polarization induced component $p_{y}$ and polarization transfer component $C_{x}$ vanish. This can be seen from the expressions for these polarization components. There are 12 independent complex amplitudes. The induced polarization $p_{y}$ and the polarization transfer $C_{x}$ are proportional to the imaginary and real parts, respectively, of the same combination of amplitudes [18]. For example, $p_{y}$ is given by

$$
f(\theta) p_{y}=2 \Im \sum_{i=1}^{3}\left[F_{i,+}^{*} F_{i+3,-}+F_{i,-} F_{i+3,+}^{*}\right]
$$

with $f(\theta)=\sum_{i=1}^{6} \sum_{ \pm}\left|F_{i, \pm}\right|^{2}$. The hadron helicity conserving amplitudes are $F_{1,+}=\left\langle\lambda_{p} \lambda_{n}|T| \lambda_{\gamma} \lambda_{d}\right\rangle=\left\langle\frac{1}{2} \frac{1}{2}|T| 11\right\rangle, F_{3,-}=\left\langle-\frac{1}{2}-\frac{1}{2}|T| 1-1\right\rangle$, and $F_{5, \pm}=\left\langle \pm \frac{1}{2} \mp \frac{1}{2}|T| 10\right\rangle$. Thus, each helicity-conserving amplitude multiplies a helicity non-conserving amplitude, and both observables vanish in case of HHC. The polarization transfer $C_{z}$ is given by 


$$
f(\theta) C_{z}=\sum_{i=1}^{6} \sum_{ \pm} \pm\left|F_{i \pm}\right|^{2}
$$

With HHC, $f(\theta) C_{z} \rightarrow F_{1,+}^{2}-F_{3,-}^{2}+F_{5,+}^{2}-F_{5,-}^{2}$, which is generally nonzero. However, at $\theta_{c m}=90^{\circ}$, relations are expected between the helicity conserving amplitudes [19]. Both $F_{1,+}$ and $F_{3,-}$ should be small, since they couple an initial state of $S=0$ or 2 to a final state of $S=1$, thus requiring orbital angular momentum effects. $F_{5,+}$ and $F_{5,-}$ should be nearly equal, since the difference between the two amplitudes is only that the proton and neutron helicities are reversed. Thus, $C_{z}$ should also approximately vanish with $\mathrm{HHC}$.

We consider now the most recently published cross section data, from Hall $\mathrm{C}$ at the Thomas Jefferson National Accelerator Facility (JLab) [1], to illustrate the variety of physical models in which explanations of photodisintegration have been attempted. Fig. 1 presents existing high energy cross section data, and partially illustrates the ambiguity in interpretation mentioned above.

- The cross sections at $90^{\circ}$ and $69^{\circ}$ follow the pQCD scaling law, although pQCD is generally not expected to describe exclusive reactions at energies of a few $\mathrm{GeV}$.

- At $90^{\circ}$ and for energies up to $1.6 \mathrm{GeV}$, the meson baryon model calculations of the Bonn group [12] (not shown) also is in very good agreement with the data.

- The asymptotic meson exchange model [5] (not shown) also generally agrees with the cross sections up to $\approx 3 \mathrm{GeV}$. The model will eventually break down, becuase it does not quite scale as $s^{-11}$. This model includes a conventional description of the deuteron plus an additional hard component, normalized to fit the data at $1 \mathrm{GeV}$. The hard component presumably results from the underlying quark degrees of freedom, and thus is perhaps better considered to be a hybrid model.
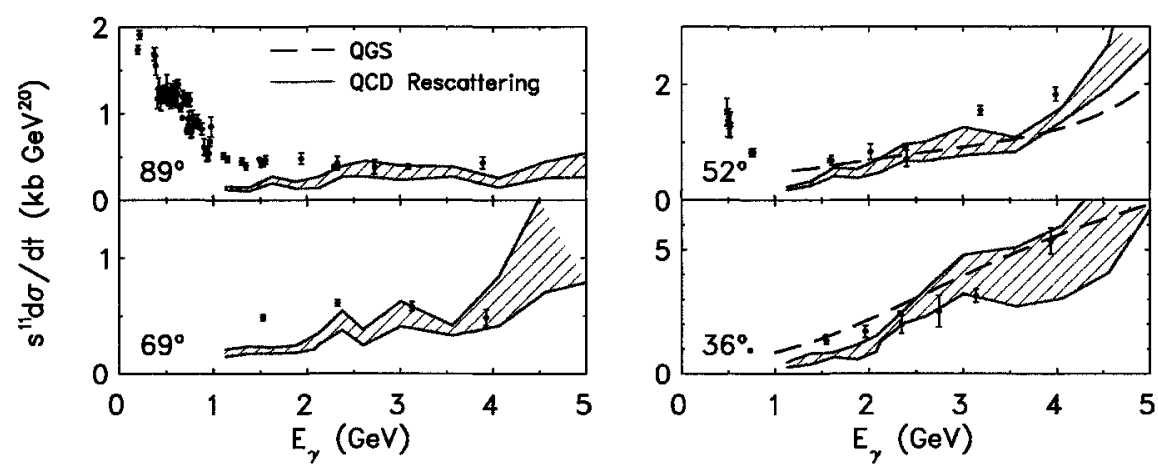

FIGURE 1. $s^{11} d \sigma / d t$ vs $E_{\gamma}$ for different center of mass angles. Data and curves are described in the text. 
- The quark gluon string (QGS) [20] model takes the reaction to be dominated by three quark exchange, which can be calculated with Regge trajectory approaches. This nonperturbative QCD model works well at small $-t$.

- The QCD rescattering model [6] is a large $-t$, large $E_{\gamma}$ nonperturbative QCD model. It calculates absolute cross sections for photodisintegration from the deuteron wave function, the hard photo-quark coupling, and the nucleonnucleon scattering amplitude, taken from nucleon nucleon data. Thus, this approach includes, through the nucleon nucleon data, soft nonperturbative physics. The resulting calculation (hatched region) describes the data well, even beyond the limits of the models validity.

- Miller [22] and Radyushkin [23] have independently shown how, in a meson baryon and in a nonperturbative quark exchange model, respectively, the cross section should more or less follow phase space factors multiplied by nucleon form factors. This behavior is not too different from pQCD, at least over a limited kinematic range. This helps explain why the data and calculations in different frameworks are similar.

\section{EXPERIMENT}

Experiment E89-019 [21] was done at JLab Hall A. Longitudinally polarized electrons were incident on a copper radiator, producing an untagged Bremsstrahlung beam of circularly polarized photons. The photons and electrons then struck a liquid deuterium target. Eight beam energies from 0.5 to $2.5 \mathrm{GeV}$ were used. Protons from the target were detected at a center of mass angles near $90^{\circ}$ in one of the Hall A high resolution spectrometers, equipped with a focal plane polarimeter (FPP). The photon energy was reconstructed from the measured proton momentum and scattering angle. Background contributions from the target windows and from electrodisintegration were subtracted. Events with final-state pions were excluded by requiring the reconstructed photon energy to be near the electron beam energy.

Polarized protons scatter in the FPP carbon analyzer with an azimuthal asymmetry. The distributions were analyzed by means of a maximum likelihood method to obtain the induced and transferred polarization components. Spin precession in the spectrometer magnetic fields and the helicity of the electron beam were taken into account.

The polarimeter calibration was checked with $e p$ elastic scattering data at each of the eight momentum settings. In $e p$ elastic scattering, the ratio of the proton electromagnetic form factors $G_{E}$ and $G_{M}$ determines the polarization transfer components, usually called $P_{l}$ and $P_{t}$. The ratio of these two components also directly determines the ratio of the form factors $G_{E} / G_{M}$, and the magnitudes determine the product of beam helicity and polarimeter analyzing power, $h A_{C}$. Here, $A_{C}$ indicates a carbon analyzer, as has been used in most previous intermediate energy polarimeters. With Møller polarimeter measurements of the beam helicity, $A_{C}$ is 


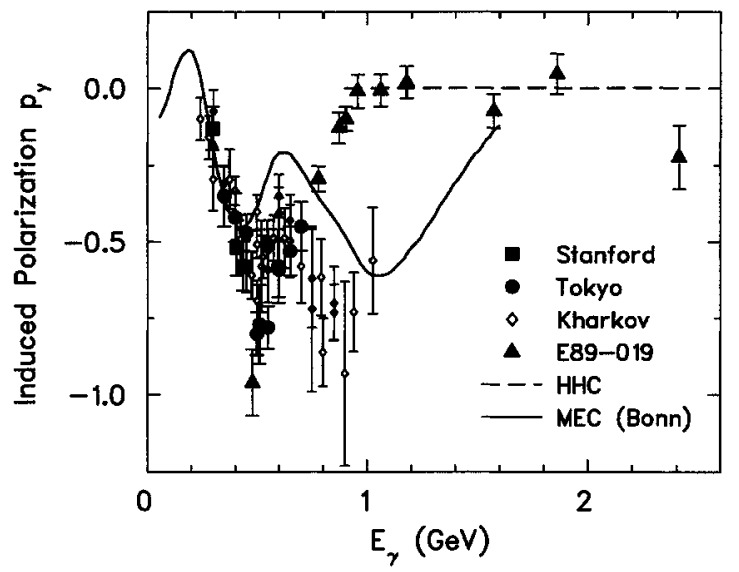

FIGURE 2. Induced polarization $p_{y}$ in the $\vec{\gamma} d \rightarrow \vec{p} n$ reaction at $\theta_{c m}=90^{\circ}$.

determined, and agrees well with previous work [24]. Note that, while $A_{C}$ does not affect the extracted form factor ratio $G_{E} / G_{M}$, it does affect the size of the uncertainty of the ratio.

Neglecting two photon exchange, the $e p$ scattering has no induced polarization; thus, it also directly measures the small instrumental asymmetries, providing small corrections to the induced polarization $p_{y}$. Uncertainty in the instrumental asymmetry model dominates the systematic uncertainties for the induced polarization, \pm 0.04 . For the polarization transfer data, the false asymmetries largely cancel with the helicity difference, leaving a smaller systematic uncertainty of about \pm 0.02 . The systematic uncertainties tend to be small, smaller than the statistical uncertainties, because the polarizations measured are generally small, and uncertainties related to the carbon analyzing power and beam polarization are multiplicative factors.

\section{RESULTS}

The preliminary result for the normal component $p_{y}$ of the induced polarization is shown as triangles in Fig. 2. Our data agree with earlier lower energy measurements from Stanford [25], Tokyo [26], and Kharkov [18,27], but disagree with the higher energy Kharkov results [28]. The higher energy Kharkov data were taken under much more difficult conditions than our measurement, which featured much more extensive calibrations, and lower backgrounds. We find that the induced polarization is small, consistent with vanishing above $E_{\gamma} \approx 1 \mathrm{GeV}$. The six highest energy data points average to $-0.02 \pm 0.02$.

The solid curve shows the result of the meson-baryon calculation of the Bonn group [12]. There is fair qualitative agreement between the calculation and the data in the $\Delta$-resonance region, but we find no evidence in our data for the predicted 
structure near $1 \mathrm{GeV}$. Instead, our $p_{y}$ data appear to support HHC (dashed curve), and thus pQCD. In the Bonn calculation the structure results largely from the $D_{13}$ and $D_{33}$ resonances interfering with the Born amplitude. Since there are only a few resonances with large photo-nucleon couplings in this energy region, the disagreement is an unexpected result, and suggests that the meson-baryon picture, at least as calculated, is not applicable.

The question is to what extent the Bonn calculation can be considered reliable and representative of meson baryon theory in general, and thus whether one can conclude anything about the general applicability of meson baryon theory. The answer is probably that no general conclusions can be made at this time. In the Bonn calculation, and others [9-11,13], final state interactions appear to have small effects on $p_{y}$. It should be noted that these models generally start from older potentials, derived largely from nucleon nucleon scattering up to $350 \mathrm{MeV}$. To match $s$, one considers nucleon nucleon scattering at a beam energy twice the photon energy; nucleon nucleon scattering at $2-5 \mathrm{GeV}$ is highly inelastic, and thus probably the final state interactions in these calculations are not under good control.

If the final state interactions are indeed small, the Born amplitude is largely real, and the resonance - Born interference generates the induced polarization. The induced polarization results from the imaginary part of an interference of amplitudes, and one expects that isolated resonances lead to an energy dependent polarization that is maximal at the peak of the resonance. Especially since polarizations are more sensitive to the resonances than are the cross sections, overlapping resonances including those with small photo-couplings will change the detailed predictions from this simple picture.

The Bonn calculation includes $\pi, \rho, \eta$, and $\omega$ exchange, plus 17 well-established nucleon and $\Delta$ resonances with mass less than $2 \mathrm{GeV}$ and $J \leq 5 / 2$, for which resonance parameters were taken from the Particle Data Group. Earlier polarization calculations [9-11], which included only the $\Delta$ resonance, have similar predictions to [12] at low energies. The inclusion of the Roper, $S_{11}$, and $D_{13}$ was studied in [13], also with similar predictions to [12]. Thus, there is qualitative agreement between several calculations and the intuitive expectation.

Given approximations in the calculations, which are not discussed, uncertainties in resonance parameters, and possible problems in final state interactions, it is clear that one cannot conclude anything generally about whether meson baryon models are in principle capable of representing the induced polarization data shown above. We speculate, however, that hybrid models such as [5] are likely to succeed, since it appears that the cross section at large energies comes largely from the hard component of the deuteron, if final state interactions generate small polarizations in this framework.

Both the scaling of the cross section and the vanishing of $p_{y}$ start near $1 \mathrm{GeV}$, and are consistent with pQCD predictions. Because of the failure of existing mesonbaryon calculations, and the surprising agreement with $\mathrm{HHC}$ in a region in which pQCD is not expected to explain the data, further tests are required. More tests 

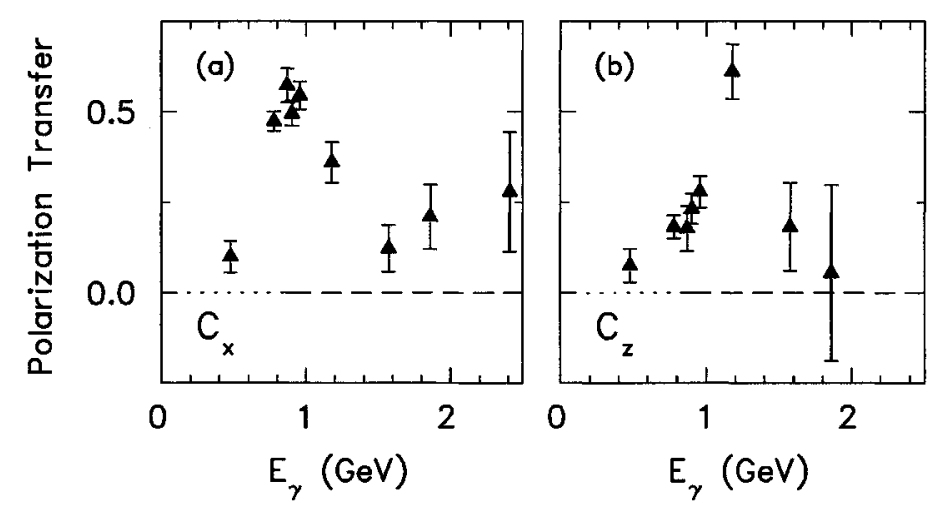

FIGURE 3. Polarization transfer in the $\vec{\gamma} d \rightarrow \vec{p} n$ reaction at $\theta_{c m}=90^{\circ}, C_{x}$ (a) and $C_{z}$ (b).

are possible with the polarization transfer observables $C_{x}$ and $C_{z}$.

Our preliminary results are shown in Fig. 3 ; neither other data nor calculations exist. While $C_{z}$ appears to approach zero near $2 \mathrm{GeV}$, the $C_{x}$ data are non-zero and indicate that hadron helicity is not conserved in this energy range. The generally accepted expectation is that pQCD leads to HHC; thus we conclude that one should not try to explain the data as arising from pQCD. While this conclusion agrees with theoretical bias [29] that perturbative processes will lead to only a small fraction of the observed strength, we note here that there is a weakness to this argument. There are both nonperturbative and perturbative corrections to the pQCD polarization prediction of $\mathrm{HHC}$ that we have shown. For example, even neglecting possible orbital angular momentum contributions [17], one expects that there are perturbative quark helicity flip contributions [16]. Including these contributions will lead to a violation of $\mathrm{HHC}$, but with a smooth approach to vanishing polarizations, which we cannot rule out.

We construct here a toy model to indicate how these observables could approach the HHC limit. Each helicity-flip should cause the amplitude to decrease faster by one power of $t$. This result is well known for the nucleon form factors, for which it is expected that $-t F_{2} / F_{1}$ approaches a constant at large $-t$. We assume the helicity conserving amplitudes have the form $F_{i}=A_{i} f(s, t)$, where $A$ is a complex magnitude and $f(s, t)$ contains the kinematic variations. The leading corrections come from single helicity flip amplitudes, that we assume to have the form $F_{j}=A_{j} f(s, t) / t$. At sufficiently large $-t$ - possibly much larger than the range in this experiment - the cross section is dominated by non-helicity-flip amplitudes, and the expression given above for $C_{x}$ can be seen to lead to a $1 / t$ behavior. Presumably $p_{y}$ vanishes in the data due to a lack of an imaginary part of the interference. With the additional assumptions of [19], $C_{z}$ should approach zero like $1 / t^{2}$. Figure 4 shows that these $t$ dependences, normalized to the data, are in good, but not compelling agreement. Exploring this possibility further requires more precise data. 

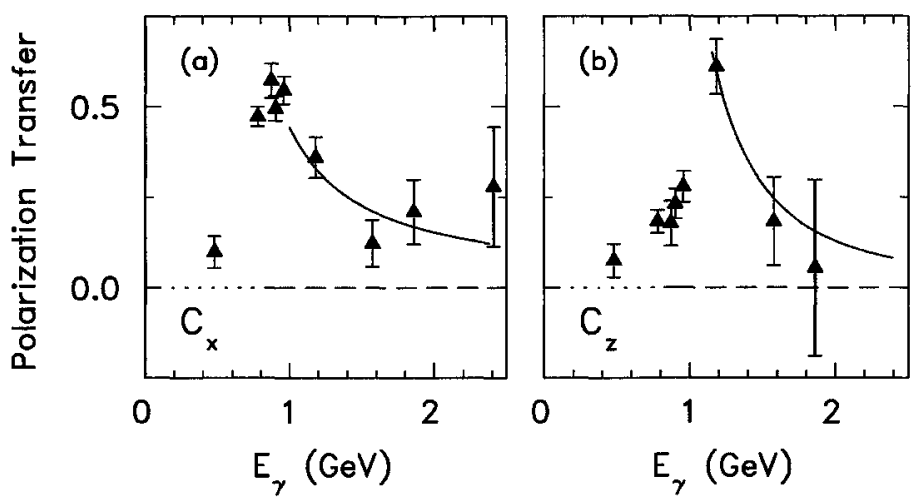

FIGURE 4. Polarization transfer in the $\vec{\gamma} d \rightarrow \vec{p} n$ reaction at $\theta_{c m}=90^{\circ}, C_{x}$ (a) and $C_{z}$ (b), compared to a toy model "prediction" of the approach to helicity conservation, normalized to the data.

We have shown above that existing MEC and $\mathrm{pQCD}$ do not predict the polarization data. We now consider several nonperturbative quark models, which have been applied to deuteron photodisintegration cross sections. The QCD rescattering model [6] provides absolute cross section calculations that agree with the data. The nonperturbative contributions are effectively included in the hard scattering $p n$ amplitude, which is taken from the $p n$ data. Preliminary induced polarization calculations [14] from this model indicate that $p_{y}$ is small, for energies above about $2.5 \mathrm{GeV}$, as it is for $E_{\gamma}$ from 1 to $2.4 \mathrm{GeV}$ in the present data. There are also cross section calculations in the quark-gluon string model [30], and polarization calculations are underway [15]. The quark exchange model [31] suggests that the amplitudes are nearly real; this can explain $p_{y}$, but calculations are needed for $C_{x}$ and $C_{z}$. The reduced nuclear amplitudes model [32] does not reproduce the cross sections well, and assumes helicity conservation, which would incorrectly predict that $C_{x}$ vanishes.

\section{CONCLUSIONS}

The induced polarization above about $1 \mathrm{GeV}$ is small, consistent with vanishing. The polarization transfers $C_{x}$ and $C_{z}$ are consistent with a steady decrease in magnitude starting slightly above $1 \mathrm{GeV}$, though the precision of the data is such that this result is not compelling.

These results have not been predicted in any theoretical approach. The induced polarization is clearly inconsistent with the only available high energy meson baryon polarization calculation. The results of this calculation are typical of what is seen in other meson baryon calculations, but one cannot rule out that an improved future calculation in this framework will agree with the data. No calculations of 
polarization transfer exist for comparison.

The vanishing of the induced polarization is naturally explained by $\mathrm{HHC}$ within pQCD, but the polarization transfer observable $C_{x}$ does not vanish. This clearly shows, with no assumptions, that $\mathrm{HHC}$ is not valid for deuteron photodisintegration in this energy region [33]. Thus, the data disagree with the usually expected behavior from $\mathrm{pQCD}$. But the data do not rule out a possible perturbative approach to $\mathrm{HHC}$, as demonstrated in a toy model.

If meson baryon theory and pQCD are both not applicable, then one is left by elimination with nonperturbative quark and hybrid models. These new benchmark data provide clear tests for the polarization calculations that will soon be available with these models.

\section{REFERENCES}

1. C. Bochna et al., Phys. Rev. Lett. 81, 4576 (1998); J.E. Belz et al., Phys. Rev. Lett. 74, 646 (1995); S.J. Freedman et al., Phys. Rev. C 48, 1864 (1993); J. Napolitano et al., Phys. Rev. Lett. 61, 2530 (1988).

2. L.C. Alexa et al., Phys. Rev. Lett. 82, 1374 (1999).

3. D. Abbott et al., Phys. Rev. Lett. 84, 5053 (2000).

4. R.G. Arnold et al., Phys. Rev. C 42, R1 (1990).

5. A.E.L. Dieperink and S.I. Nagorny, Phys. Lett. B 456, 9 (1999).

6. L.L. Frankfurt et al., Phys. Rev. Lett. 84, 3045 (2000); L.L. Frankfurt et al., Nucl. Phys. A 663, 349 (2000).

7. S.J. Brodsky and G.R. Farrar, Phys. Rev. Lett. 31, 1153 (1973).

8. R. Holt, Phys. Rev. C 41, 2400 (1990).

9. J.M. Laget, Nucl. Phys. A 312, 265 (1978).

10. W. Liedemann and H. Arenhövel, Nucl. Phys. A 465, 573 (1987).

11. H. Tanabe and K. Ohta, Phys. Rev. C 40, 1905 (1989).

12. Y. Kang et al., Abstracts of the Particle and Nuclear Intersections Conference, (MIT, Cambridge, MA 1990); Y. Kang, Ph.D. dissertation, Bonn (1993).

13. M. Schwamb, H. Arenhövel, P. Wilhelm, Few Body Syst. 19, 121 (1995).

14. M.M. Sargsian, private communication.

15. E. De Sanctis, L.A. Kondratyuk, private communications.

16. See S.J. Brodsky and G.P. Lepage, Phys. Rev. D 24, 2848 (1981), and references therein.

17. T. Gousset, B. Pire, and J.P. Ralston, Phys. Rev. D 53, 1202 (1996).

18. V.P. Barranik et al., Nucl. Phys. A 451, 751 (1986).

19. D. Sivers, private communication; S.I. Nagornyi, Yu.A. Kasatkin, and I.K. Kirichenko, Yad. Fiz. 55, 345 (1992) [Sov. J. Nucl. Phys. 55, 189 (1992)].

20. L.A. Kondratyuk et al., Phys. Rev. C 48, 2491 (1993).

21. JLab Experiment E89-019, R. Gilman, R.J. Holt, and Z.-E. Meziani, spokespersons; K. Wijesooriya et al., submitted to Phys. Rev. Lett.

22. G.A. Miller, private communication.

23. A. Radyushkin, private communication. 
24. B. Bonin et al., Nucl. Instrum. Methods A 288, 379 (1990); M.W. McNaughton et al., Nucl. Instrum. Methods A 241, 435 (1985).

25. F.F. Liu et al., Phys. Rev. 165, 1478 (1968).

26. T. Kamae et al., Phys. Rev. Lett. 38, 468 (1977); T. Kamae et al., Nucl. Phys. B 139, 394 (1978); H. Tkeda et al., Phys. Rev. Lett. 42, 1321 (1979); H. Ikeda et al., Nucl. Phys. B 172, 509 (1980).

27. A.S. Bratashevskij et al., Nucl. Phys. B 166, 525 (1980); A.S. Bratashevskij et al., Yad. Fiz. 31, 860 (1980) [Sov. J. Nucl. Phys. 31, 444 (1980)]; A.S. Bratashevskij et al., Pis'ma Zh. Eksp. Teor. Fiz. 35, 489 (1982) [JETP Lett. 35, 605 (1982)]; A.S. Bratashevskij et al., Yad. Fiz. 43, 785 (1986) [Sov. J. Nucl. Phys. 43, 499 (1986)]; A.A. Zybalov et al., Nucl. Phys. A 533, 642 (1991); V.B. Ganenko et al., Z. Phys. A 341, 205 (1992).

28. A.S. Bratashevskij et al., Pis'ma Zh. Eksp. Teor. Fiz. 34, 410 (1981); A.S. Bratashevskij et al., Pis'ma Zh. Eksp. Teor. Fiz. 36, 174 (1982) [JETP Lett. 36, 216 (1982)]; A.S. Bratashevskij et al., Yad. Fiz. 44, 960 (1986) [Sov. J. Nucl. Phys. 44, $619(1986)]$.

29. N. Isgur and C. Llewellyn Smith, Nucl. Phys. B 317, 526 (1989); A. V. Radyushkin, Nucl. Phys. A 523, 141c (1991).

30. E. De Sanctis et al., Few Body Syst. Suppl. 6, 229 (1992); L. A. Kondratyuk et al. Phys. Rev. C 48, 2491 (1993).

31. A. Radyushkin, private communication.

32. S.J. Brodsky and J.R. Hiller, Phys. Rev. C 28, 475 (1983).

33. Note also recent $\Sigma$ asymmetry results from Yerevan: F. Adamian et al., Eur. Phys. J. A 8, 423 (2000). 